\title{
Electric and dielectric properties of solution-gas interface-grown PbS films
}

\author{
P S NIKAM and K A PATHAN* \\ PG Department of Physical Chemistry and *Department of Physics, MSG College, \\ Malegaon Camp 423 105, India
}

MS received 16 November 1993; revised 15 February 1994

\begin{abstract}
Dielectric properties and $I-V$ characteristics of solution-gas interface-formed $\mathrm{PbS}$ thin-film capacitors ( $\mathrm{Al} / \mathrm{PbS} / \mathrm{Al}$ ) of various thicknesses have been studied in the frequency range $10-10^{6} \mathrm{~Hz}$ at various temperatures $(300-443 \mathrm{~K})$. Current-voltage $(I-V)$ characteristics show space-charge-limited conduction. Dielectric constant $(\varepsilon)$ increases with increasing film thickness and temperature and decreases with increase of frequency. The loss factor $(\tan \delta)$ peaks observed in $\tan \delta$ vs frequency and $\tan \delta$ vs temperature reveal relaxation effect from dipolar orientation. These maxima shift to higher-temperature region with increasing frequency. The latge increase in capacitance $(C)$ and dielectric constant $(\varepsilon)$ towards lowfrequency $(f)$ region indicates the possibility of an interfacial polarization mechanism in this region.
\end{abstract}

Keywords. $I-V$ characteristics; space-charge-limited current; capacitance; loss factor; relaxation effect; dipolar orientation; interfacial polarization.

\section{Introduction}

Thin films of $\mathrm{PbS}$ find applications in the manufacture of $\mathrm{Q}$ switches for lasers and for solar control coatings for window glazing (Nair et al 1990). However, films deposited by thermal means are extremely fragile, porous and can only be successfully deposited at very low evaporation rates. Therefore chemically and mechanically stable $\mathrm{PbS}$ films are formed using chemical deposition technique. However, previous studies of $\mathrm{PbS}$ films formed by bath deposition have been restricted only to their photoconductivity (Acharya and Bose 1971, 1979; Burger et al 1971; Espvik et al 1971; Blount et al 1973; Kothiyal et al 1976, 1980). The following is the only report of investigation of the electric and dielectric behaviour of $\mathrm{PbS}$ films formed at the interface of aqueous solution of $\mathrm{Pb}\left(\mathrm{NO}_{3}\right)_{2}$ and $\mathrm{H}_{2} \mathrm{~S}$ gas.

\section{Experimental}

One hundred $\mathrm{ml}$ of $1 \% \mathrm{~Pb}\left(\mathrm{NO}_{3}\right)_{2}$ aqueous solution in a dish was exposed to $\mathrm{H}_{2} \mathrm{~S}$ gas atmosphere. A PbS film was formed on the surface of $\mathrm{Pb}\left(\mathrm{NO}_{3}\right)_{2}$ solution due to chemical reaction taking place between cations $\left(\mathrm{Pb}^{2+}\right)$ from the solution and anions $\left(\mathrm{S}^{2-}\right)$ from the gas. This film was picked up on clean glass substrate with vacuumevaporated pure aluminium $(99.999 \%)$ film of thickness $\sim 2200 \AA$ as base electrode. $\mathrm{PbS}$ film was allowed to float in distilled water many times in order to remove impurities in the film. The film was dried in vacuum. Pure aluminium was again evaporated (thickness $\sim 2200 \AA$ ) on $\mathrm{PbS}$ film to form sandwich-type $\mathrm{Al} / \mathrm{PbS} / \mathrm{Al}$ capacitor (figure 1) completing MIM structure. The effective area of capacitor ranged between 0.6 and $1 \mathrm{~cm}^{2}$. These capacitors were stabilized by self-annealing for several 


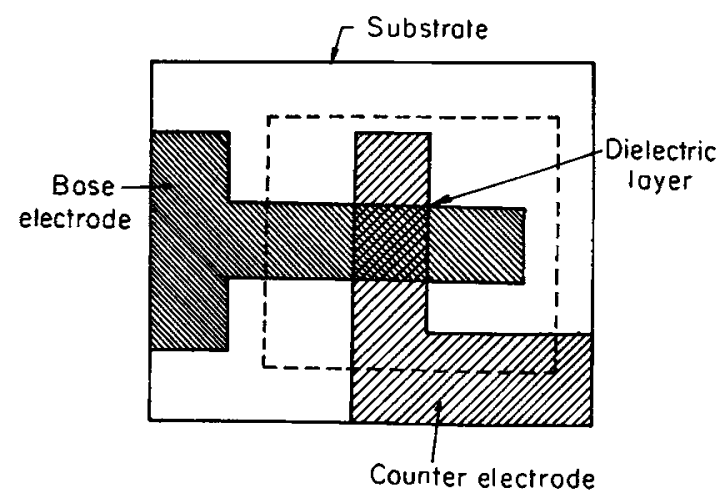

Figure 1. Structure of the capacitor.

days in vacuum followed by thermal annealing in vacuum at $10^{-5}$ torr at $130^{\circ} \mathrm{C}$ for $8 \mathrm{~h}$. The dielectric, thickness, resistivity, $I-V$ characteristics, capacitance $(C)$ and loss factor $(\tan \delta)$ of $\mathrm{PbS}$ films of different thicknesses were measured in vacuo $\left(10^{-5}\right.$ torr) at different temperatures $(300-443 \mathrm{~K})$ and frequencies $\left(f=10-10^{6} \mathrm{~Hz}\right)$ in the manner described previously (Nikam and Aher 1993; Nikam and Pathan 1993). Capacitance was measured in ohmic region.

\section{Results and discussion}

Variation of $\log R$ with reciprocal of absolute temperature $T$ showed semiconducting behaviour of $\mathrm{PbS}$ films. Energy of activation $(\Delta E)$ values (table 1) decrease with increase of film thickness $(d)$, which could be explained by the cases explained earlier (Nikam and Aher 1993).

Figure 2 shows the variation of $\log$ current $(I)$ vs log voltage $(V)$ at $300 \mathrm{~K}$ for films of different thickness. These curves are analysed with the help of a model proposed by Rose (1955) for defect insulators containing shallow traps. According to this model, space-charge-limited current (SCLC) is directly proportional to $V^{2}$ and inversely to $d^{3}$, which in fact was observed in the present investigation. It has also been found that voltage at which the transition from ohmic to SCL conduction occurs $\left(V_{\text {trans. }}\right)$ is directly proportional to $d^{2}$ (figure 3 ), which is the criterion for SCL conduction (Lampert 1956; Lampert et al 1959). The energy level $(\sim 0.054 \mathrm{eV})$ of traps to which the charge carriers are injected from the electrodes does not vary with the applied field and contact remains injecting all the time, which is a prerequisite (Lamb 1967) for SCL currents.

Thickness dependence of capacitance $(C)$ and dielectric constant $(\varepsilon)$ is represented in figure 4. Capacitance decreases with rise of film thickness as expected while dielectric constant initially increases rapidly and then attains a constant value of $\sim 6$ at $d \sim 2400 \AA$.

Dielectric constant $(\varepsilon)$, capacitance $(C)$, thickness $(d)$, area $(A)$ of the dielectric film and free space permittivity $\left(\varepsilon_{0}\right)$ are interrelated by the equation (Peek et al 1985).

$$
C=\frac{\varepsilon \varepsilon_{0} A}{d}
$$


Table 1. Variation of energy of activation $(\Delta E)$ with thickness $(d)$ of PbS films.

\begin{tabular}{lccc}
\hline & $\begin{array}{l}\Delta E \\
(\mathrm{eV})\end{array}$ & $\begin{array}{c}\Delta \\
(\AA)\end{array}$ & $\begin{array}{c}\Delta E \\
(\mathrm{eV})\end{array}$ \\
& from resistance & $(\AA)$ & from capacitance \\
\hline 2201 & 0.220 & 1912 & 0.192 \\
2481 & 0.206 & 2316 & 0.142 \\
2793 & 0.180 & 2550 & 0.121 \\
2812 & 0.174 & 2910 & 0.103 \\
3134 & 0.102 & 3210 & 0.094 \\
\hline
\end{tabular}

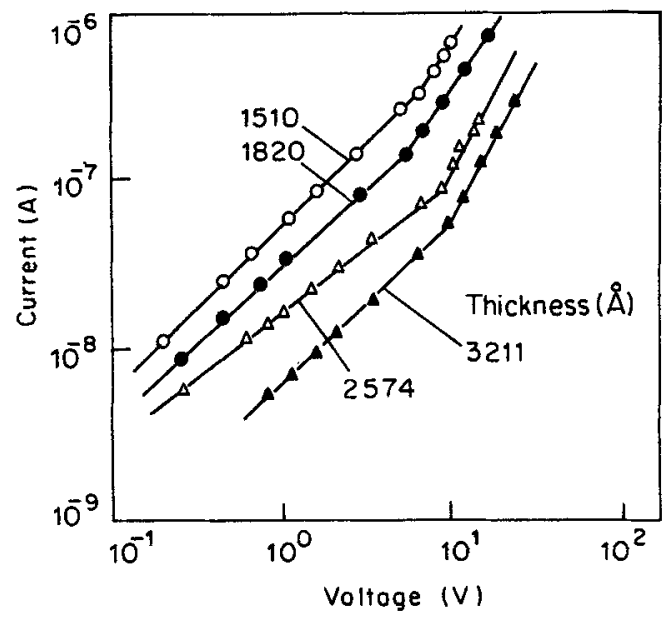

Figure 2. $\log I$ vs $\log V$ at $300 \mathrm{~K}$ for different thicknesses of $\mathrm{PbS}$ films.

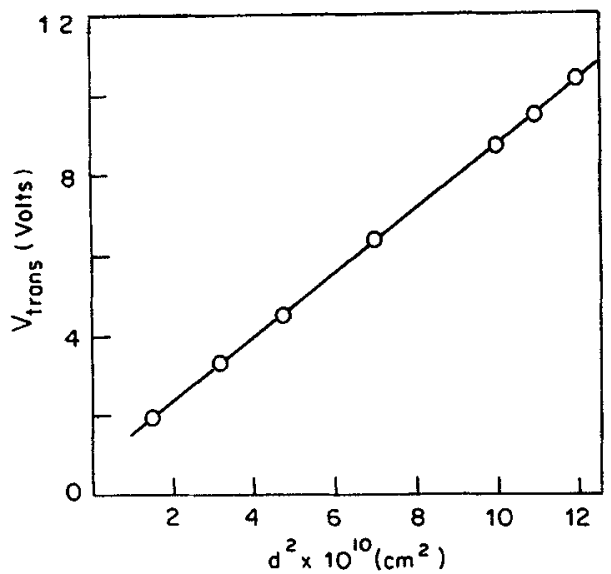

Figure 3. $V_{\text {trans. }}$ vs thickness squared. 
The dielectric constant decreases rapidly in lower-thickness region, probably due to a contribution to the capacitance from a thin oxide layer present on the lower aluminium electrode (Nikam and Pathan 1993). Alternatively the variation of $\varepsilon$ with film thickness for thinner films (figure 2) appears to be related to the voids and discontinuities present in them. Capacitors made out of such films may then be considered in the idealized cases to comprise many capacity elements (Goswami and Dharmadhikari 1982) consisting of three types of dielectric media, namely (i) $\mathrm{PbS}$ film, (ii) air from voids and (iii) oxide layer on lower Al base electrode in thinner film regions. For very thin films, dielectric constant will tend to be unity, corresponding to dielectric constant of air. The effective $\varepsilon$ of the total capacity elements will hence

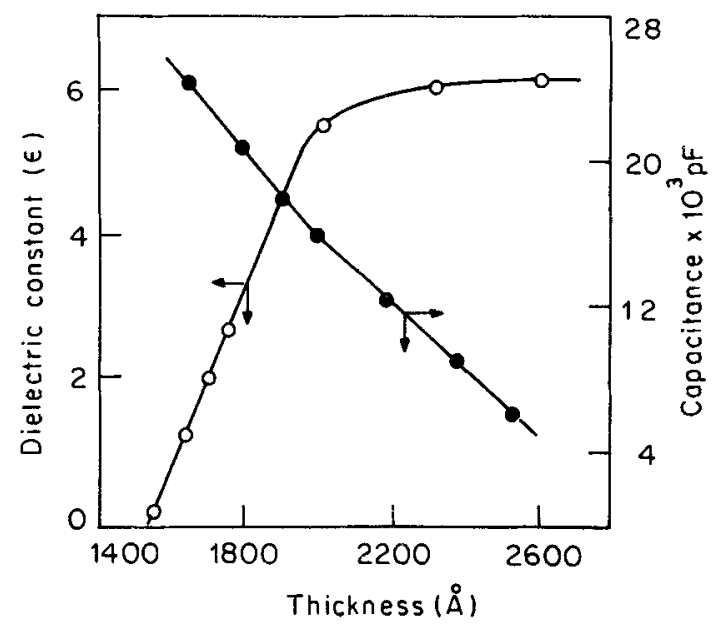

Figure 4. Capacitance and dielectric constant vs thickness of the film.

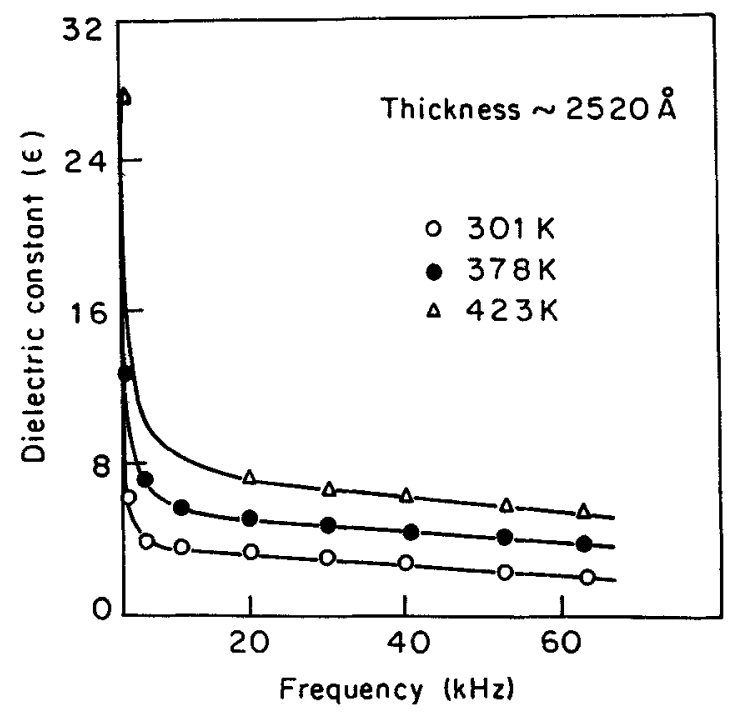

Figure 5. Dielectric constant vs frequency for a film of thickness $\sim 2520 \AA$. 
depend on the amounts of voids, aluminium oxide, etc. present in the capacitor films. Thus with the increase in film thickness voids and aluminium oxide will continually be reduced and hence $\varepsilon$ will increase, eventually attaining the constant value. The mechanism of the film growth process explains at least qualitatively the variation of $\varepsilon$ with $d$ observed by us.

The variation of dielectric constant with frequency at various temperatures is presented in figure 5 for $\mathrm{PbS}$ film of thickness $\sim 2520 \AA$. The dielectric constant decreases with frequency at all temperatures but this variation is less pronounced as the temperature decreases. This can be attributed to an interfacial polarization caused by space charge and macroscopic field distortion (Von Hippel et al 1953). The contribution from space charge polarization is mainly noticeable in low frequency region, and depends on the purity and perfection of the films.

The loss peak observed in $\tan \delta$ vs frequency (figure 6) curve reveals a relaxation effect in $\mathrm{PbS}$ films. The shift in the loss peak towards the higher-frequency region with increasing temperature is in accordance with the Debye theory of dipole orientation (Debye 1929). For a Debye process at higher temperatures and for a given frequency, a maximum in $\tan \delta$ will occur at a temperature such that $2 \pi f_{\text {relax }}=(1 / \tau)$, where $\tau$ is the relaxation time. Accordingly, $f_{\text {relax }}$ will be greater when $\tau$ is less. The rise in temperature causes a reduction in the mean time of stay of ionic dipoles (Wert 1950 ), which in turn causes $\tau$ to decrease and $f_{\text {relax }}$ to occur in the higher audiofrequency range as observed in the present study. Sulphur deficiency (Landry and Langley 1979) may cause the formation of dipoles in the film structure.

The temperature dependence of dielectric constant for a film of thickness $\sim 2873 \AA$

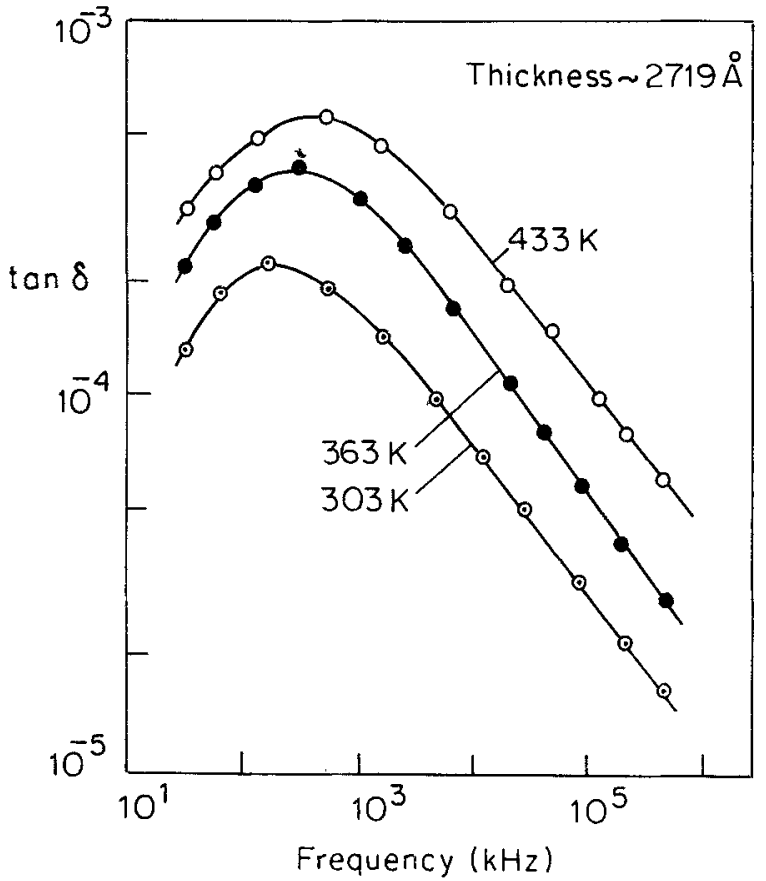

Figure 6. Tan $\delta$ vs frequency for a film of thickness $\sim 2719 \AA$. 


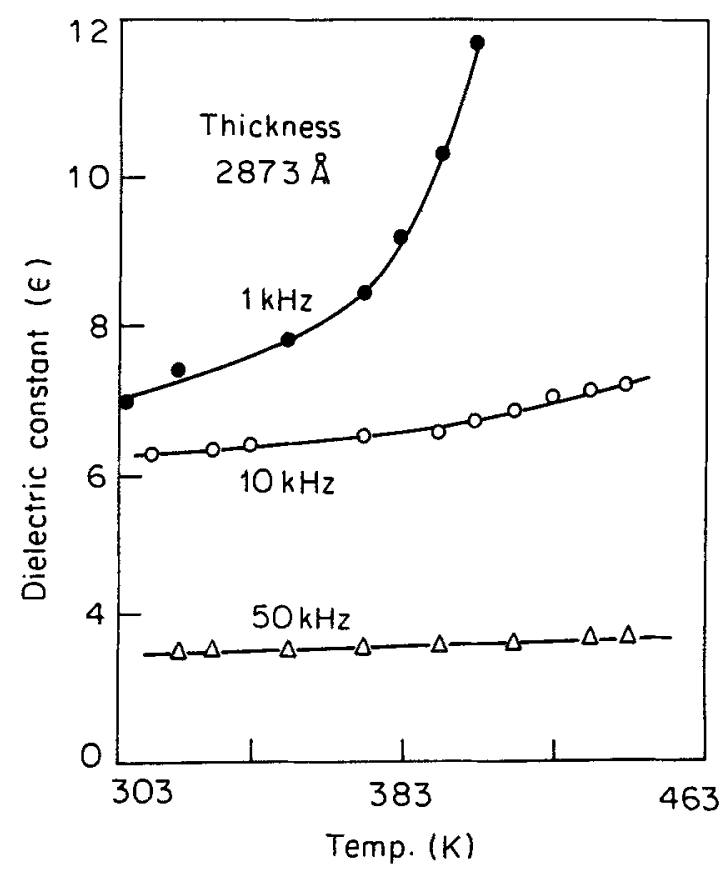

Figure 7. Dielectric constant vs temperature for a film of thickness $\sim 2873 \AA$.

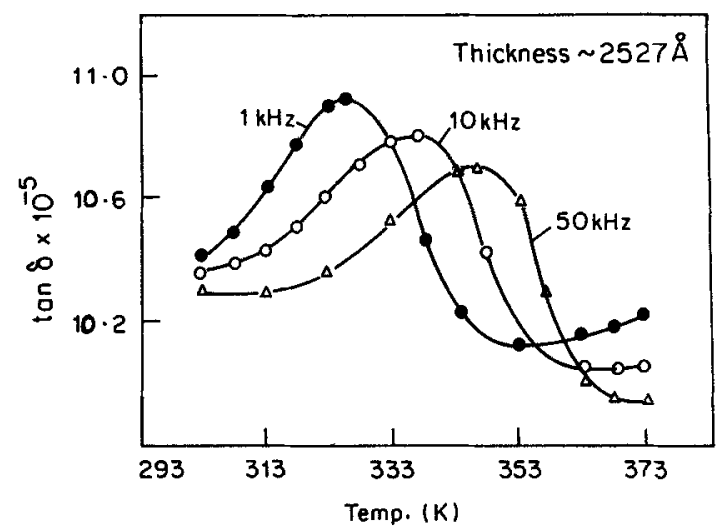

Figure 8. Tan $\delta$ vs temperature for a film of thickness $\sim 2527 \AA$.

at 1,10 and $50 \mathrm{kHz}$ has been represented in figure 7 . The increase in dielectric constant with increase of temperature above room temperature may be partly due to the expansion of the lattice (Beamo 1965) and partly due to the excitation of charge carriers from imperfection sites.

Figure 8 shows the variation of loss factor $(\tan \delta)$ with temperature for a film of thickness $\sim 2527 \AA$ at 1,10 and $50 \mathrm{kHz}$ frequencies. All these curves show maxima. The maxima shift to higher-temperature region with increasing frequency. The presence of maxima in $\tan \delta$ vs temperature curves reveals a dielectric relaxation effect in $\mathrm{PbS}$ films arising from dipolar orientation. 


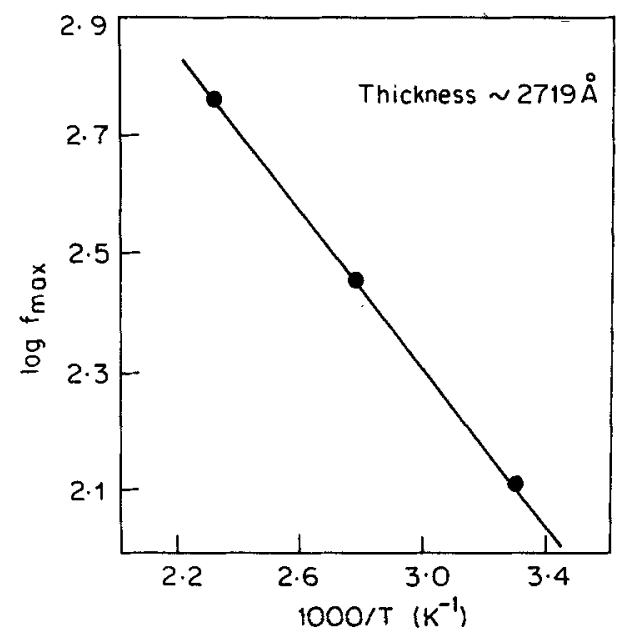

Figure 9. $\log f_{m}$ vs $1 / T$ for a film of thickness $\sim 2719 \AA$.

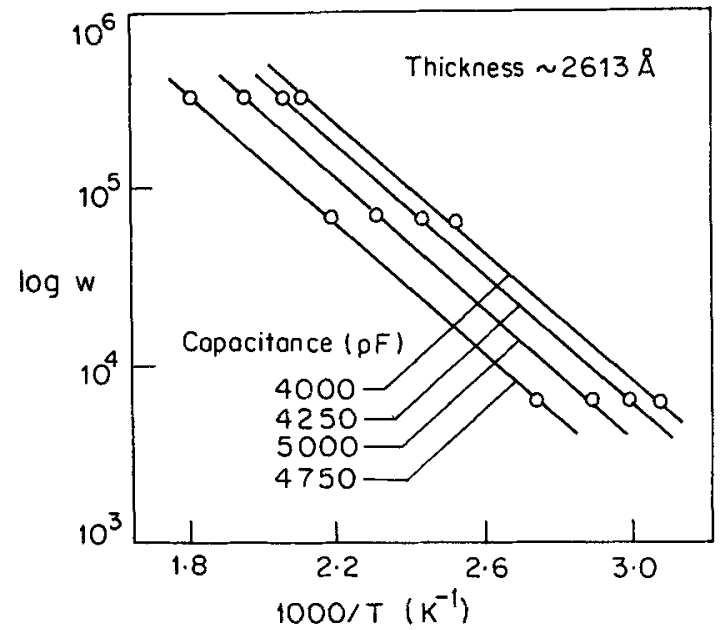

Figure 10. $\log \omega$ vs $1 / T$ for four constant capacitances of film of thickness $\sim 2613 \AA$.

The variation of dielectric constant $(\varepsilon)$ with frequency and temperature is similar to that of capacitance with frequency and temperature.

Making use of the relation (Nadkarni and Simmons 1970; Paramasivan et al 1980)

$$
f_{m}=f_{0} \exp \left(\frac{-\Delta E}{k T}\right),
$$

a plot is made (figure 9) of the logarithm of the frequency $f_{m}$ at which $\tan \delta$ is a maximum against the reciprocal absolute temperature. The slope of this curve yields an activation energy of $\sim 0.1294 \mathrm{eV}$ for a film of thickness $\sim 2719 \AA$.

According to Simmons et al (1970), for any capacitor system with an inherent 
resistance, $R_{0}$,

$$
\omega=A R_{0} \exp \left(\frac{-\Delta E}{k T}\right),
$$

where $\omega$ is the angular frequency $(\omega=2 \pi f)$ and $A$ is a constant whose magnitude depends on the particular selected value of constant capacitance. The variation of $\log \omega$ with $1 / T$ for four constant capacitances for a film of thickness $\sim 2613 \AA$ is represented in figure 10 . The four plots are found to be linear and parallel. Activation energies of $\mathrm{PbS}$ films thus obtained are included in table 1 for comparison with those calculated from resistance measurements. The activation energy in table 1 indicates that $\Delta E$ from $\varepsilon$ measurements is lower than $\Delta E$ from $R$ measurements. It may be due to instrumental errors.

Therefore, it is concluded that the $\mathrm{PbS}$ films formed by using solution-gas interface technique are semiconducting in nature, exhibiting SCL conduction. The measurements of dielectric properties of these films indicate the presence of dielectric relaxation effect arising from dipolar orientation.

\section{Acknowledgement}

Authors are grateful to Mr S S Patil, Principal, M S G College, for providing laboratory facilities.

\section{References}

Acharya H N and Bose H N 1971 Phys. Stat. Sol. (a) 6K 43

Acharya H N and Bose H N 1979 Indian J. Pure Appl. Phys. A53 6

Beamo W R 1965 Electronics of solids (New York: McGraw-Hill) p. 385

Blount G H, Schriber P J, Smith D K and Yamada R T 1973 J. Appl. Phys. 44978

Burger P A, Malan O G, Kunzo O A and Fink W 1971 Z. Naturforsch. 26132

Debye P 1929 Polar molecules (New York: Dover)

Espvik S, Chem-Ho-Wu and Bube R H 1971 J. Appl. Phys. 423513

Goswami A and Dharmadhikari V S 1982 Thin Solid Films 87119

Kothiyal G P, Deshpande R Y and Ghose B 1976 Proc. Nucl. Phys. Solid State Phys. Symp. 19(C) 115

Kothiyal G P, Ghose B and Deshpande R Y 1980 J. Phys., D. Appl. Phys. 13869

Lamb D R 1967 Electrical conduction mechanism in thin insulating films (London: Methuen)

Lampert M A 1956 Phys. Rev. 1031648

Lampert M A, Rose A and Smith R W 1959 Phys. Chem. Solids 8484

Landry M J and Langley R A 1979 Thin Solid Films 67171

Nadkarni G S and Simmons J G 1970 J. Appl. Phys. 41545

Nair P K, Ocampo M, Fernandez A and Nair M Ts 1990 Solar Energy Mater. 20235

Nikam P S and Aher H S 1993 Indian J. Pure Appl. Phys. 3179

Nikam P S and Pathan K A 1993 Indian J. Pure Appl. Phys. (in press)

Paramasivan K R, Radhakrishnan M and Balsubramanian C 1980 Thin Solid Films 74189

Peek D, Guerra V C and Sladek R J 1985 J. Appl. Phys. 574803

Rose A 1955 Phys. Rev. 971538

Simmons J G, Nadkarni G S and Lancaster M C 1970 J. Appl. Phys. 41538

Von Hippel A, Gross E P, Telstis F G and Geller A 1953 Phys. Rev. 91568

Wert C A 1950 Phys. Rev. 79601 